\title{
Profile of adult erythroderma in hospitals in Lomé (Togo): study of 147 cases
}

\section{Julienne Noude Teclessou ${ }^{1,2}$, Sefaco Abla Akakpo,,2, Gmakéyi Monsila', Abas Mouhari-Toure ${ }^{3,4}$, Bayaki Saka ${ }^{1,2}$, Koussake Kombate ${ }^{1,2}$, Palokinam Pitche ${ }^{1,2}$}

${ }^{1}$ Department of Dermatology and Venerology, Teaching Hospital of Lomé, Lomé, Togo, ${ }^{2}$ Department of Health Sciences, University of Lomé, Lomé, Togo, ${ }^{3}$ Department of Dermatology, Teaching Hospital of Kara, Kara, Togo, ${ }^{4}$ Department of Health Sciences, University of Kara, Kara, Togo

Corresponding author: Dr. Julienne Noude Teclessou, E-mail: tjulie06@yahoo.fr

\begin{abstract}
Objective: The aim of this study was to describe the epidemiological and clinical profile of erythroderma in hospital setting in Lomé (Togo). Methods: We reviewed the epidemiological and clinical data of adults patients diagnosed with erythroderma in dermatology departments over a 20-year period (1997 through 2016). Results: In total, one hundred and forty seven patients were diagnosed with erythroderma during the study period ( $0.4 \%$ of all consulted patients). The average age at the onset of the disease was 41.1 years with a male: female ratio of $1.5: 1$. The most frequent cause of erythroderma was exacerbation of preexisting dermatoses (80.8\%), including eczema (84.3\%) and psoriasis (11.7\%). No cause could be identified in forty one cases (27.9\%). Apart from erythema and scaling, that were present in all patients, clinical findings were dominated by pruritus (62.33\%), fever (8.2\%), and altered general condition (8.8\%). Treatment was symptomatic and etiologic in all patients. The evolution was favorable in $47.62 \%$ of the cases, and one death occurred in a patient with cutaneous lymphoma. Conclusion: This study outlines that erythroderma is rare in Lomé (Togo). Our series had a high percentage of erythroderma secondary to preexisting dermatoses and idiopathic cases.
\end{abstract}

Key words: Erythroderma; Adult; Lomé (Togo)

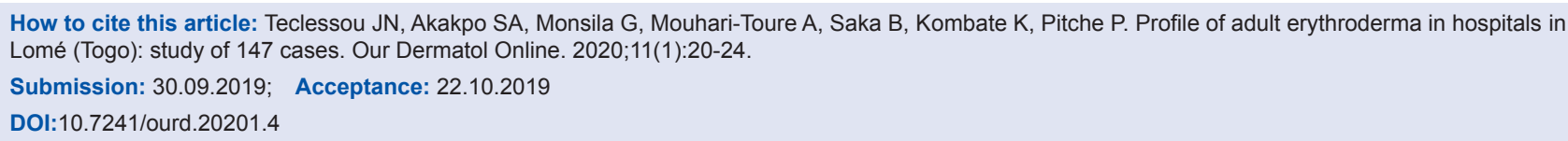




\title{
Profil des érythrodermies de l'adulte en milieux hospitalier à Lomé: étude de 147 cas
}

\section{Julienne Noude Teclessou',2, Sefaco Abla Akakpo,,2, Gmakéyi Monsila', Abas Mouhari-Toure ${ }^{3,4}$, Bayaki Saka ${ }^{1,2}$, Koussake Kombate ${ }^{1,2}$, Palokinam Pitche ${ }^{1,2}$}

\author{
${ }^{1}$ Department of Dermatology and Venerology, Teaching Hospital of Lomé, Lomé, Togo, ${ }^{2}$ Department of Health Sciences, \\ University of Lomé, Lomé, Togo, ${ }^{3}$ Department of Dermatology, Teaching Hospital of Kara, Kara, Togo, ${ }^{4}$ Department of \\ Health Sciences, University of Kara, Kara, Togo
}

Corresponding author: Dr. Julienne Noude Teclessou, E-mail: tjulie06@yahoo.fr

\section{RÉSUMÉ}

Objectif: Le but de cette étude était de décrire le profil épidémiologique, clinique, et évolutif des érythrodermies en milieu hospitalier à Lomé. Méthode: Il s'est agi d'une étude rétrospective descriptive portant sur les dossiers des patients âgés de plus de 15 ans ayant été suivi pour une érythrodermie dans les services publics de dermatologie à Lomé de janvier 1997 à décembre 2016. Résultats: Au total, 147 patients ont été inclus dans l'étude soit une fréquence de $0,4 \%$ des motifs de consultation. Lâge moyen des patients était de 4l,1ans et le sex-ratio $(\mathrm{H} / \mathrm{F})$ de 1,5. Le prurit était présent chez $62,33 \%$ des patients, une fièvre et une altération de l'état général chez respectivement $8,2 \%$ et 8,8\% des patients. La majorité (80,8\%) des patients présentaient une érythrodermie sèche. Sur le plan étiologique, les dermatoses érythrodermiques $(69,4 \%)$ étaient au premier plan, suivi des causes idiopathiques $(27,9 \%)$. Les principales dermatoses érythrodermiques étaient l'eczéma $(84,3 \%)$ et le psoriasis $(11,76 \%)$. Le traitement était symptomatique et étiologique dans certains cas. Lévolution était favorable dans $47,62 \%$ des cas, et un décès était survenu chez un patient ayant un lymphome cutané. Conclusion: Les érythrodermies constituent une affection rare en consultation dermatologique à Lomé. Les étiologies les plus fréquentes restent les dermatoses érythrodermiques.

Mots-clés: Érythrodermies; Adulte; Lomé

\section{INTRODUCTION}

Lérythrodermie ou dermatose exfoliatrice est un syndrome associant un érythème confluant touchant plus de $90 \%$ de la surface corporelle, une desquamation et une évolution prolongée [1]. C'est une urgence dermatologique pouvant mettre en jeu le pronostic vital des patients. Il s'agit d'un syndrome d'installation aigue ou subaiguë et qui implique une recherche étiologique. Les causes des érythrodermies varient en fonction de l'âge. Chez l'adulte, plusieurs circonstances peuvent être à l'origine de la survenue d'une érythrodermie notamment les dermatoses érythrodermiques ou les toxidermies. En Afrique du Nord, les érythrodermies représentaient $0,3 \%$ des motifs de consultations en dermatologie [2]. Peu d'études ont porté sur les érythrodermies en Afrique sub-Sahara [3].

Le but de notre étude était de documenter le profil épidémiologique, clinique et évolutif des érythrodermies de l'adulte en consultation dermatologique à Lomé.

\section{MATERIALS AND METHODS}

Il s'agit d'une étude rétrospective descriptive ayant concerné les services de dermatologie des Centre Hospitalier Universitaire (CHU) Sylvanus Olympio et Campus, et du service de dermatologie du Centre

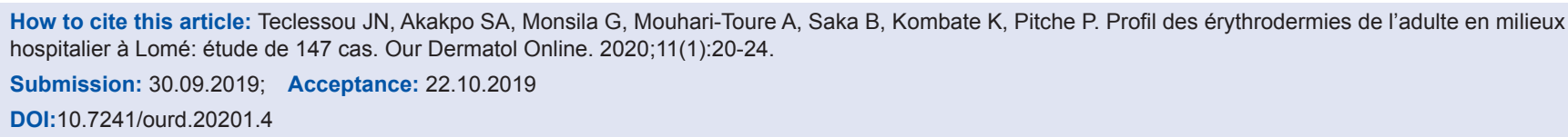


de Gbossimé à Lomé. Les dossiers des patients vus dans les différents services du ler Janvier 1997 au 31 décembre 2016 ont été revus. Les patients âgés de plus de 15 ans chez qui le diagnostic d'érythrodermie était retenu ont été inclus. Etait considéré comme érythrodermie, les patients ayant un érythème diffus, touchant plus de $90 \%$ de la surface corporelle avec squames et d'évolution prolongée. Les toxidermies à type de nécrolyse épidermique toxique (ou syndrome de Lyell) étaient exclues de cette étude. Une fiche d'enquête à été pré établie précisant les données sociodémographiques (âge, sexe, profession, antécédents pathologiques et/ou antécédents d'érythrodermie des patients); cliniques (signes fonctionnels, signes généraux, aspects cliniques, étiologies) et paracliniques. Lanalyse des données est réalisée avec le logiciel Epi info7. La signification statistique a été définie comme $\mathrm{p}<0,05$. Nous avons utilisé le test exact de Fisher et le test non paramétrique de Kruskal-Wallis pour comparer les moyennes.

\section{Ethics Statement}

Le protocole d'étude a été soumis et a été approuvé par le comité de bioéthique du ministre de la Santé et des centres hospitaliers concernés par l'étude. Les chefs des différents services concernés par l'étude ont donnés leurs accords pour l'exploitation des dossiers.

\section{RESULTS}

Durant la période d'étude, 147 (0,4\%) des 37567 patients âgés de plus de 15 ans ont consultés pour une érythrodermie, ce qui correspond à 8 cas en moyenne par an. Lâge moyen des patients était de $41,1 \pm 15$ (extrêmes: 17 et 80 ans) et le sex-ratio $(\mathrm{H} / \mathrm{F})$ de 1,5. Les patients ayant une érythrodermies étaient en général des adultes jeunes avec une prédominance de la tranche de 26 ans à 35 ans 49 patients (33,33\%). Lérythrodermie était de survenue tardive chez les hommes (âge moyen $=45,1$ ans; extrêmes: 19 et 80 ans) que chez les femmes (âge moyen $=34,9$ ans; extrêmes 17 et 80 ans), $\mathrm{p}=0,0001$

La majorité des patients $(80,9 \%)$ présentaient une érythrodermie sèche. Le prurit était le signe fonctionnel le plus fréquent chez nos patients $(71,4 \%)$. Une fièvre (supérieure à $38^{\circ} \mathrm{C}$ ) et une altération de l'état général était retrouvées chez respectivement $12(8,16 \%)$ et $13(8,84 \%)$ des patients. Un oedème des extrémités était noté chez $23(15,64 \%)$ des patients. L'hyperkératose palmo-plantaire et les dystrophies unguéales à type d'hyperkératose sous unguéale, mélanonychie ou onycholyse étaient retrouvées chez respectivement chez $11(7,5 \%)$ et $12(8,2 \%)$ des patients et des adénopathies périphériques étaient observées chez 19 (12,9\%) des patients.

Sur le plan paraclinique, la vitesse de sédimentation était accélérée chez 47 des 61 patients l'ayant réalisée. Une anémie et une hyperleucocytose étaient notées chez respectivement 29 et 38 des 61 patients ayant réalisé une numération formule sanguine. Seul $11(7,5 \%)$ patients avaient bénéficié d’une biopsie cutanée. Tous les cas de lymphomes cutanés étaient confirmés par histologie.

Sur le plan étiologique, les dermatoses érythrodermiques étaient les étiologies les plus fréquentes avec $102(69,4 \%)$ suivies des causes idiopathiques $(27,9 \%)$. Parmi les dermatoses érythrodermiques, l'eczéma

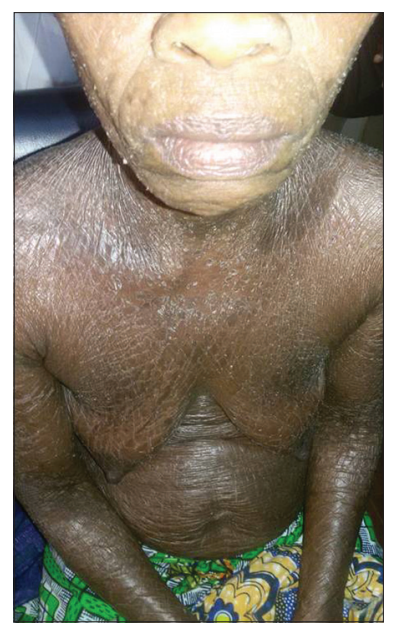

Figure 1: Erythroderma due to eczema.

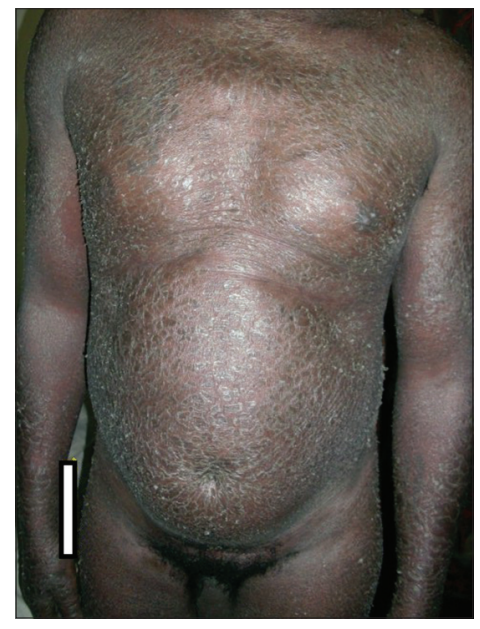

Figure 2: Erythroderma due to psoriasis in an adult. 


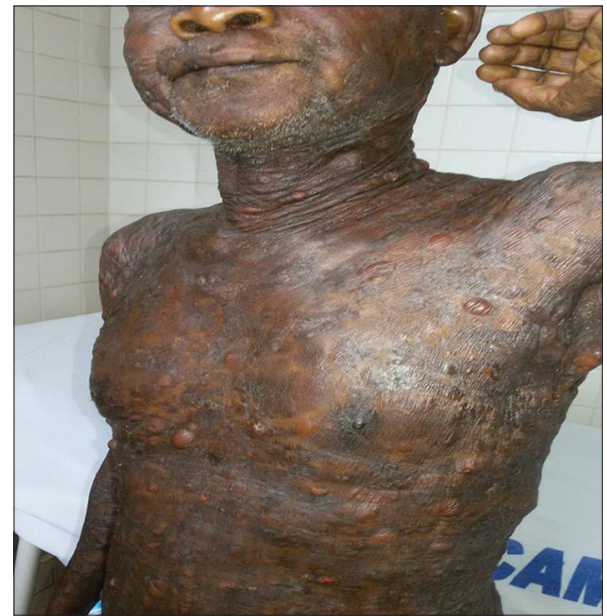

Figure 3: Erythroderma during fungus mycosis in a 62-year-old patient.

\begin{tabular}{lcc}
\multicolumn{2}{l}{ Tableau 1: Etiologies des érythrodermies } \\
\hline Etiologies & $\mathbf{n}$ & $\%$ \\
\hline Eczémas érythrodermiques & 86 & 58,50 \\
Psoriasis érythrodermiques & 12 & 8,16 \\
Lichen plan idiopathique & 3 & 2,04 \\
Pemphigus & 1 & 0,68 \\
Causes idiopathiques & 41 & 27,89 \\
Toxidermie (DRESS) & 1 & 0,68 \\
Mycosis fongoïde & 2 & 1,37 \\
Syndrome de Sézary & 1 & 0,68 \\
Total & 147 & 100 \\
\hline
\end{tabular}

érythrodermique 86 (84,3\%) (Fig. 1) et le psoriasis érythrodermique 12 (11,8\%) (Fig. 2) étaient les plus fréquentes (Tableau 1). Sur les 86 patients atteints d'un eczéma érythrodermique, 8 (9,3\%) avaient un antécédent personnel d'atopie dont 7 cas d'asthme et 1 cas de rhinite allergique. Les patients ayant un eczéma érythrodermique étaient relativement plus jeune que les patients ayant une autre étiologie d'érythrodermie (âge moyen $=39,03$ ans) $(p=0,002)$. Aucune association significative n'est notée entre le sexe et les étiologies des érythrodermies, $\mathrm{p}=0,93$

Les lymphomes cutanés comme cause d'érythrodermie dans notre étude étaient le mycosis fongoïde $(1,37 \%)$ (Fig. 3) et le Syndrome de Sézary $(0,68 \%)$. Un cas de DRESS syndrome était retrouvé chez une fille de 21 ans infectée par le VIH sous traitement antirétroviral. La molécule incriminée était l’Abacavir.

La prise en charge de l'érythrodermie avait nécessité une hospitalisation chez 18 (12,2\%) des patients. La durée moyenne d'hospitalisation était de 8 jours avec des extrêmes de 5 et 32 jours. Lévolution était favorable chez $69(47,6 \%)$ patients et $76(51,7 \%)$ étaient perdus de vue au cours du suivi. Un décès était survenu chez un patient ayant un lymphome cutané. L'évolution était favorable dans $44 \%$ des cas d'érythrodermies idiopathiques.

\section{DISCUSSION}

Nous avons enregistré environ 8 cas d'érythrodermie par an, ce qui est compris entre les 6,3 cas et 9,4 cas par an rapportés respectivement en Tunisie et au Portugal [4,5]. Les érythrodermies constituent donc une affection rare en milieu hospitalier à Lomé puisqu'elles ne représentent que $0,4 \%$ des motifs de consultation. Lâge moyen des patients était de 4l, lans et la tranche d'âge de 26 ans à 35 ans était prédominante. Ceci signe une prédominance de l'affection chez les sujets jeunes dans notre étude contrairement à l'étude de César et al. et de Hulmani et al. ou l'affection était plus fréquente dans la $6^{\text {ème }}$ décade $[5,6]$. Nous avons noté une prédominance masculine des érythrodermies avec une sex-ratio de 1,53. Cette prédominance masculine des érythrodermies à été également rapportée dans plusieurs études avec une sex-ratio variant entre 1,8 et $2,2[2,7]$.

D’une façon générale, les dermatoses érythrodermiques $(69,4 \%)$ étaient les étiologies les plus fréquentes chez l'adulte. Les dermatoses érythrodermiques ont été rapportées comme cause fréquente des érythrodermies par plusieurs auteurs [5,8-10] avec une fréquence allant de 33,3\% à 74,4\%. Cependant, les eczémas érythrodermiques sont au premier plan des dermatoses érythrodermiques dans notre étude $(84,32 \%)$ suivi du psoriasis $(11,76 \%)$ contrairement en Afrique du Nord [4], en Inde et en Chine où le psoriasis érythrodermique était la dermatose érythrodermique la plus fréquentes $(30,5 \%$ à 55,0\%) [5,6,8-11]. La rareté des psoriasis érythrodermiques dans notre étude peut s'expliquer par sa faible prévalence hospitalière faible $(0,62 \%)$ de l'affection en Afrique subsaharienne [12].

Les érythrodermies due à une toxidermie médicamenteuse étaient peut fréquente dans notre étude $(0,7 \%)$. Les érythrodermies secondaires à une toxidermie représentaient respectivement 16,6\% et $18,4 \%$ des étiologies des érythrodermies en Inde et au Brésil $[5,6]$. Ceci est due au fait que nous avons exclut de notre étude les nécroses épidermiques toxique qui ne répondent plus à la définition actuelle des érythrodermies [13].

Aucune cause n'était identifiée chez environ un quart $(27,9 \%)$ de nos patients. Cette valeur est proche de 
celle de Vasconcellos et al. (29,2\%) au Portugal [11]. Par contre elle est nettement supérieure à celle de l'étude de Yuan et al. $(6,1 \%)$ en Chine [10]. Le taux élevé des érythrodermies idiopathiques dans cette étude pourrait aussi s'expliquer par le faible taux de réalisation des examens anatomopathologiques dans notre étude (10 biopsies réalisées sur les 147 cas).

Lévolution était favorable dans $47,6 \%$ des cas et $1(0,7 \%)$ patient était décédé. Li et al avaient également noté un faible taux de décès ( 5 sur 260 patients) [8]. Ces données montrent que dans la majorité des cas les dermatoses érythrodermiques (majorité des étiologiques) n’engagent pas le pronostic vital à court terme.

\section{CONCLUSION}

Les érythrodermies constituent une affection rare en consultation dermatologique à Lomé. Il s'agit d'affections retrouvées plus fréquemment chez les adultes jeunes de sexe masculin. Les principales causes de ces érythrodermies chez les adultes étaient les dermatoses érythrodermiques dont principalement l'eczéma érythrodermique suivi du psoriasis érythrodermique.

\section{ACKNOWLEDGEMENTS}

- Lérythrodermie est un syndrome cutané peu fréquent en Afrique subsaharienne.

- Peu d'étude ont rapportées les aspects épidémiologiques et cliniques des érythrodermies.

\section{Statement of Human and Animal Rights}

All procedures followed were in accordance with the ethical standards of the responsible committee on human experimentation (institutional and national) and with the Helsinki Declaration of 1975, as revised in 2008.

\section{Statement of Informed Consent}

Informed consent was obtained from all patients for being included in the study.

\section{REFERENCES}

1. Puzenat E, Humbert J-M, Bonnet blanc B, Crickx J-C R. Erythrodermie. Ann Dermatol Venereol. 2002;129(10 suppl):2S207-9.

2. Rym BM, Mourad M, Bechir Z, Dalenda E, Faika C, Iadh AM, et al. Erythroderma in adults: A report of 80 cases. Int J Dermatol. 2005;44:731-5.

3. Ndiaye B, Sissoko F, Strobel M, Marchand J. Les érythrodermies de l'adulte (à propos de 77 cas à Dakar). Dakar Médical. 1979;24:65-74.

4. Khaled A, Sellami A, Fazaa B, Kharfi M, Zeglaoui F, Kamoun MR. Acquired erythroderma in adults: a clinical and prognostic study. J Eur Acad Dermatol Venereol. 2010;24:781-8.

5. César A, Cruz M, Mota A, Azevedo F. Erythroderma. A clinical and etiological study of 103 patients. J Dermatol Case Rep. 2016;10:1-9.

6. Hulmani M, Nandakishore B, Bhat MR, Sukumar D, Martis J, Kamath G, et al. Clinico-etiological study of 30 erythroderma cases from tertiary center in South India. Indian Dermatol Online J. 2014;5:25-9.

7. Akhyani M, Ghodsi ZS, Toosi S, Dabbaghian H. Erythroderma: A clinical study of 97 cases. BMC Dermatol. 2005;5:5.

8. Li J, Zheng HY. Erythroderma: a clinical and prognostic study. Dermatology. 2012;225:154-62.

9. Pal S, Haroon TS. Erythroderma: a clinico-etiologic study of 90 cases. Int J Dermatol. 1998;37:104-7.

10. Yuan XY, Guo JY, Dang YP, Qiao L, Liu W. Erythroderma: A clinicaletiological study of 82 cases. Eur J Dermatol. 2010;20:373-7.

11. Vasconcellos C, Domingues PP, Aoki V, Miyake RK, Sauaia N, Martins JE. Erythroderma: Analysis of 247 cases. Rev Saude Publica. 1995;29:177-82.

12. Somé-Korsaga N, Ouédraogo D-D, Yadieu PK, Ouédraogo MS, Tapsoba GP, Ilboudo L, et al. Profil sociodémographique et anatomoclinique du psoriasis en milieu hospitalier et tropical à Ouagadougou, Pan African Med J. 2016;23:110.

13. Lachapelle JM. Erythrodermie. In: Saurat JH, Lipsker D, Thomas L, Borradori L. Dermatologie et maladies sexuellement transmissibles. Paris: Masson, 2017: 282-286.

Copyright by Boubacar Julienne Noude Teclessou, et al. This is an open access article distributed under the terms of the Creative Commons Attribution License, which permits unrestricted use, distribution, and reproduction in any medium, provided the original author and source are credited.

Source of Support: Nil, Conflict of Interest: None declared. 